\title{
Adsorption of anionic polyelectrolyte and comb polymers onto lead magnesium niobate
}

\author{
Aylin Şakar-Deliormanlı ${ }^{\mathrm{a}, *}$, Erdal Çelik ${ }^{\mathrm{b}}$, Mehmet Polat $^{\mathrm{a}}$ \\ a İzmir Institute of Technology, Chemical Engineering Department, Urla, İzmir, Turkey \\ ${ }^{\mathrm{b}}$ Dokuz Eylul University, Metallurgy and Materials Engineering Department, Izmir, Turkey
}

Received 16 April 2007; received in revised form 20 August 2007; accepted 3 September 2007

Available online 7 September 2007

\begin{abstract}
This paper presents the results concerning the adsorption mechanism of polyacrylic acid (PAA) and polyacrylic acid/polyethylene oxide (PAA/PEO) comb polymer onto lead magnesium niobate (PMN) powders. In the study adsorption behavior of PAA and PAA/PEO onto PMN surface were determined in aqueous solutions and the influence of $\mathrm{pH}$ and ionic strength was investigated. Results showed that adsorption of PAA or PAA/PEO increased with decreasing $\mathrm{pH}$ of the suspensions. The increase in the ionic strength or the presence of divalent cations caused an increase in the adsorption of both polyelectrolytes. It was observed that the adsorption reaches a maximum when PAA is fully complexed in solution. On the other hand, the increase in the adsorption of PAA/PEO onto PMN in the presence of monovalent or divalent salt was attributed to the decrease in the electrostatic forces rather than complex formation with the divalent metal ions in solution. Turbidity measurements showed that there is no complex formation between the divalent metal ions and PAA/PEO comb polymers due to shielding effect of the PEO teeth.
\end{abstract}

(C) 2007 Elsevier B.V. All rights reserved.

Keywords: Adsorption; Comb polymers; PAA; Lead magnesium niobate; Perovskites

\section{Introduction}

Lead magnesium niobate (PMN) is a relaxor ferroelectric material which is characterized by a diffuse phase transition over a broad temperature range and a frequency dependent maximum in its relative dielectric permittivity [1]. It has many potential applications such as multilayer ceramic capacitors, actuators and electro-optic devices [2]. It also finds applications in motors, pumps, optical scanning systems, and vibration isolators [3].

The manufacturing of multilayer electronic devices composed of perovskite materials such as PMN generally involves a colloidal process which requires preparation of stable suspensions. Organic additives such as polyelectrolytes play a significant role in preparation of stable suspensions because they offer an effective means of modifying particle-particle interactions [4]. When adsorbed on inorganic surfaces, these species

\footnotetext{
* Corresponding author. Tel.: +90 232 7506697; fax: +90 2327506645.

E-mail address: aylindeliormanli@iyte.edu.tr (A. Şakar-Deliormanlı).
}

impart electrostatic, steric or electrosteric stabilization. Polyelectrolyte adsorption is highly dependent on the electrostatic interactions between the polyelectrolyte and the surface; hence, the surface chemistry of the solid phase and the solution properties of the polyelectrolyte are important parameters, regulated by the $\mathrm{pH}$ and the ionic strength [5].

The polyacrylic acid (PAA) is a widely used polyelectrolyte in ceramic systems [6-9]. It contains ionizable carboxylic acid groups along its backbone. These groups dissociate and form negatively charged carboxylate groups at high $\mathrm{pH}$. However, PAA dispersant systems are less effective in high ionic strength suspensions especially those containing multivalent ions. Because multivalent ions interfere with the electrostatic repulsive barrier and cause flocculation [9].

On the other hand perovskite materials having the general chemical formula, $\mathrm{ABO}_{3}$ such as $\mathrm{PMN}$ are composed of a cation or more than one cation at the A site that is generally soluble in water and a cation at the B site that is only slightly soluble over the most of the $\mathrm{pH}$ ranges [10]. Therefore, the leaching of lead magnesium niobate in water is a major issue in colloidal processing. Accordingly, there is a need for new alternative dis- 
persants that can induce stabilization for suspensions containing multivalent ions.

Previously Kirby and Lewis studied the effect of polyacrylic acid/polyethylene oxide (PAA/PEO) comb polymers on the dispersion of aqueous cement systems and barium titanate suspensions [9,11]. Whitby et al. [12] investigated the interactions between silica particles in the presence of PAA/PEO comb polymers. Similarly Deliormanlı et al. [13] showed that PAA/PEO comb polymers are effective dispersants for PMN/water system. These polymers impart stability to PMN suspensions over a wide $\mathrm{pH}$ range and ionic strength where pure PAA fails. Because presence of PEO teeth shields the PAA backbone from ion interactions [9]. However, adsorption mechanism of PAA/PEO comb polymers onto PMN surface was not investigated yet. Therefore, the aim of the current study was to analyze the adsorption behavior of PAA/PEO comb polymer onto PMN surface in aqueous medium and investigate the effect of $\mathrm{pH}$ and ionic strength on the adsorption. As a benchmark further experiments were performed using pure polyacrylic acid (PAA).

\section{Experimental}

\subsection{Materials}

Lead magnesium niobate, $\mathrm{Pb}\left(\mathrm{Mg}_{1 / 3} \mathrm{Nb}_{2 / 3}\right) \mathrm{O}_{3}$ powder which was produced by combustion spray pyrolysis method provided by Praxair Specialty Ceramics, Woodinville, WA, USA. Powder purity is $99.9 \%$ as reported by the manufacturer. Bulk density (Helium pycnometer Micromeritics 1330, Norcross, GA, USA), and the BET surface area (Model ASAP 2400, Micromeritics, Norcross, GA, USA) of the powder are measured to be $7.967 \mathrm{~g} / \mathrm{cm}^{3}$ and $1.168 \mathrm{~m}^{2} / \mathrm{g}$, respectively. Particle size distribution of the powder was obtained using a particle size analyzer (Model CAPA-700, Horiba Ltd., Tokyo, Japan) and the $d_{50}$ of the powder was measured to be $2.03 \mu \mathrm{m}$.

Adsorption behavior of polyacrylic acid graft polyethylene oxide comb polymers PAA/PEO (W.R. Grace and Co., Connecticut, Cambridge, MA) were tested in the study. These comb polymers consist anionic PAA backbone with ionizable carboxylic acid group $\left(\mathrm{COO}^{-}\right)$per monomer unit and charge neutral PEO teeth grafted onto the backbone at random intervals through ester linkages [9]. They are produced by co-polymerization method as reported by the manufacturer company. Fig. 1 shows the chemical structure of the pure PAA and PAA/PEO comb polymers used in this study. In order to investigate the effect of comb polymer molecular architecture on the

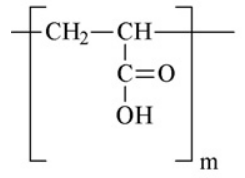

(a)

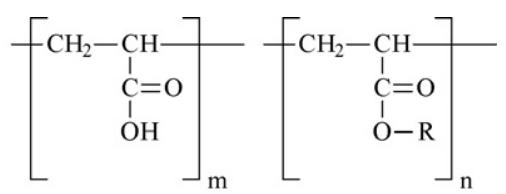

(b)
Fig. 1. Chemical structure of the (a) PAA and the (b) PAA/PEO comb polymers (Note: $\mathrm{PEO}$ teeth, $\mathrm{CH}_{2} \mathrm{CH}_{2} \mathrm{O}$ ) $\mathrm{x}-\mathrm{CH}_{3}$ ), adapted from Kirby and Lewis [11].

adsorption behavior comb polymers were tested having different molecular weight, carboxylic acid to ester linkage ratio. For comparison purposes experiments were also performed using pure PAA (Polyscience Inc., Warrington, PA). Table 1 shows the properties of the comb polymers and PAA used in the study.

\subsection{Method}

\subsubsection{Suspension preparation}

Lead magnesium niobate suspensions were prepared at different solids loadings, in the absence and presence of PAA/PEO comb polymers and pure PAA. After PMN powder was added to the stock solution of polymeric dispersant, the suspension was ultrasonicated (F550 Sonic Dismembrator, Fisher Scientific, Pardubice, Czech Republic) for $2.30 \mathrm{~min}$ using a $1 \mathrm{~s}$ on/off pulse sequence. The $\mathrm{pH}$ was adjusted using $1 \mathrm{M}$ solutions of $\mathrm{HNO}_{3}$ and $\mathrm{NH}_{4} \mathrm{OH}$. Suspensions were magnetically stirred for $24 \mathrm{~h}$ at room temperature prior to measurements.

\subsubsection{Zeta potential measurements}

Zeta potential measurements were performed using a zeta sizer (Malvern 3000 HS, Malvern Instruments Ltd., Worcestershire, United Kingdom) with aqueous dip cell apparatus whose electrodes were properly cleaned before each measurement to avoid contamination. The device makes use of the laser Doppler microelectrophoresis technique for the zeta potential determination. The zeta potential of particles was calculated from their electrophoretic mobility by means of the Henry correction of the Smoluchowski equation. Since this technique allows measurements only in dilute suspensions, samples were diluted prior to measurement using sedimentation and then a small amount of sediment redispersed with the supernatant. The measurements were periodically checked against a calibration standard with a zeta potential of $-50 \mathrm{mV}( \pm 5)$. Experiments were performed in triplicate, and the results given are the average of 10 measurements.

Table 1

Properties of the PAA and PAA/PEO comb polymers used in the study

\begin{tabular}{|c|c|c|c|c|}
\hline & PAA Mw (g/mol) & PEO Mw (g/mol) & Acid:ester & Theoretical Mw (g/mol) \\
\hline Pure PAA & 5000 & - & - & 5000 \\
\hline PAA/PEO360 & 5000 & 360 & $3: 1$ & 13,583 \\
\hline PAA/PEO400 & 5000 & 400 & $3: 1$ & 14,537 \\
\hline PAA/PEO1000 & 5000 & 1000 & $3: 1$ & 22,850 \\
\hline PAA/PEO1000 & 5000 & 1000 & $7: 1$ & 15,300 \\
\hline PAA/PEO2000 & 5000 & 2000 & $7: 1$ & 25,600 \\
\hline
\end{tabular}




\subsubsection{Adsorption behavior}

The adsorption amount of PAA and PAA/PEO comb polymers on PMN powder surface in aqueous suspensions was determined by total organic carbon (TOC) analyzer (Shimadzu TOC-V CPH, Japan), which provided a quantitative measure of the non-adsorbed fraction of such species in solution. Suspensions with a solids loading of $10 \mathrm{vol} \%$ were prepared by adding an appropriate amount of PMN powder to an aqueous stock solutions of PAA and comb polymer with varying concentration. Upon mixing for $24 \mathrm{~h}$, the suspensions were centrifuged at $4000 \mathrm{rpm}$ for $60 \mathrm{~min}$. The supernatant was used for TOC analysis. Several aliquots of each sample were measured, and an average value was reported based on standard calibration curves.

\subsubsection{Turbidity measurements}

Dilute polymer solutions $(5 \mathrm{mg} / \mathrm{ml})$ were prepared by adding an appropriate amount of PAA or PAA/PEO comb polymer to deionized water. The $\mathrm{pH}$ was adjusted using either a $0.1 \mathrm{M}$ $\mathrm{HNO}_{3}$ or $0.1 \mathrm{M} \mathrm{NaOH}$ solution. These polymer solutions were magnetically stirred for $0.5 \mathrm{~h}$ to allow for equilibration. One molar solutions of $\mathrm{KCl}, \mathrm{MgCl}_{2}$ were prepared by dissolving $\mathrm{KCl}$ and $\mathrm{MgCl}_{2} \cdot 6 \mathrm{H}_{2} \mathrm{O}$ (Fisher Chemicals, Fair Lawn, $\mathrm{NJ}$ ) in deionized water. These salt solutions were used to vary the monovalent, divalent salt concentration of the polymer solutions. The turbidity of dilute polymer solutions of varying $\mathrm{pH}$ and salt concentration was measured by a turbidimeter (WTW 555, Germany). Measurements were performed using nephelometric ratio method and results were recorded in terms of NTU with \pm 2 of value or \pm 0.01 NTU accuracy.

\section{Results and discussion}

\subsection{Electrokinetic properties}

Fig. 2 shows the variation of isoelectric point (IEP) of PMN as a function of solids loading. The shift in the IEP of PMN powder to lower $\mathrm{pH}$ values at low solid concentrations can be explained based on the solubility considerations. Previously, Deliormanlı et al. investigated the dissolution of $\mathrm{Pb}^{2+}$ and $\mathrm{Mg}^{2+}$ ions from $\mathrm{PMN}$ surface in water. Results showed that $\mathrm{Pb}^{2+}$ and $\mathrm{Mg}^{2+}$ ion

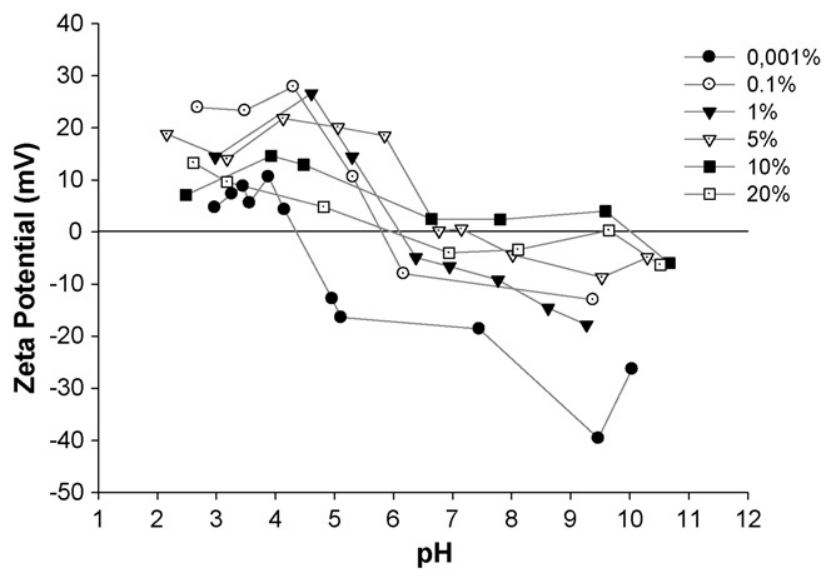

Fig. 2. The IEP of PMN suspensions at various solids loadings (vol\%) [14].

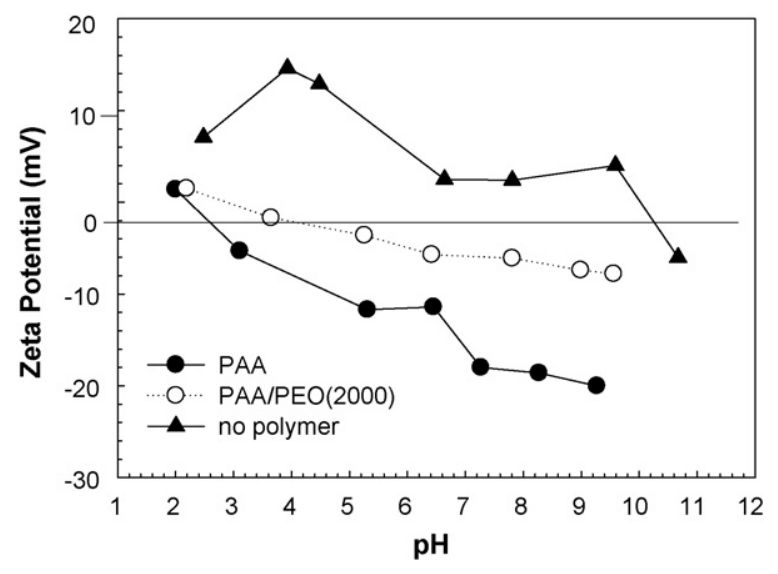

Fig. 3. The IEP of PMN suspensions in the presence of PAA $\left(1.1 \mathrm{mg} / \mathrm{m}^{2}\right)$ and PAA/PEO $\left(4.29 \mathrm{mg} / \mathrm{m}^{2}\right)$. Solids loading: $10 \mathrm{vol} \%$.

dissolution increased with decreasing $\mathrm{pH}$. On the other hand, $\mathrm{Nb}^{5+}$ showed very low dissolution under the same conditions [14]. Therefore, when the PMN is suspended in water a surface layer develops around the particle due to ion dissolution. The surface layer on PMN particles in acidic region is thicker in dilute suspensions compared to the concentrated suspensions. Because the rate of surface layer growth in concentrated suspensions is inhibited by the mass transfer. The surface layer around the PMN increases surface acidity of the powder. Therefore, the IEP of the powder decreases as the surface layer increases [14-16].

Fig. 3shows the effect of PAA and PAA/PEO on the IEP of aqueous PMN suspensions at $10 \mathrm{vol} \%$. The IEP of bare PMN suspension at $10 \mathrm{vol} \%$ in the absence of polyelectrolyte was around $\mathrm{pH} 10$ (Fig. 2). On the other hand, addition of PAA $\left(1.075 \mathrm{mg} / \mathrm{m}^{2}\right.$ of PMN) or PAA/PEO comb polymer $\left(4.29 \mathrm{mg} / \mathrm{m}^{2}\right.$ of PMN) to the system caused a shift in the IEP to a lower $\mathrm{pH}$ value. This may be explained by the adsorption of negatively charged PAA or PAA/PEO onto positively charged PMN surface. Additionally, presence of carboxylic groups on the polymer shift the IEP as a result of complexation reaction between these groups and the cationic species on the particle surface.

It is known that polyelectrolyte adsorption may lead to an overcompensation of the surface charge and thus to an inversion of the surface charge. According to the previous study of Joanny, this is always the case in the limit of weak screening where the Debye screening length is larger than the thickness of the adsorbed polymer layer $[17,18]$.

\subsection{Adsorption behavior by TOC}

Adsorption behavior PAA and PAA/PEO comb polymers onto PMN surface was studied as a function of polyelectrolyte concentration, $\mathrm{pH}$ and ionic strength. All experiments were performed at a solids loading of $10 \mathrm{vol} \%$. The $\mathrm{pH}$ dependence of adsorption isotherms of PAA and PAA/PEO onto PMN surface are shown in Figs. 4 and 5, respectively. The plots represent the amount of polymer that is adsorbed per unit surface area of the powder versus the total amount of polymer that is added to the suspension. The dash line denotes the complete $(100 \%)$ adsorption. 


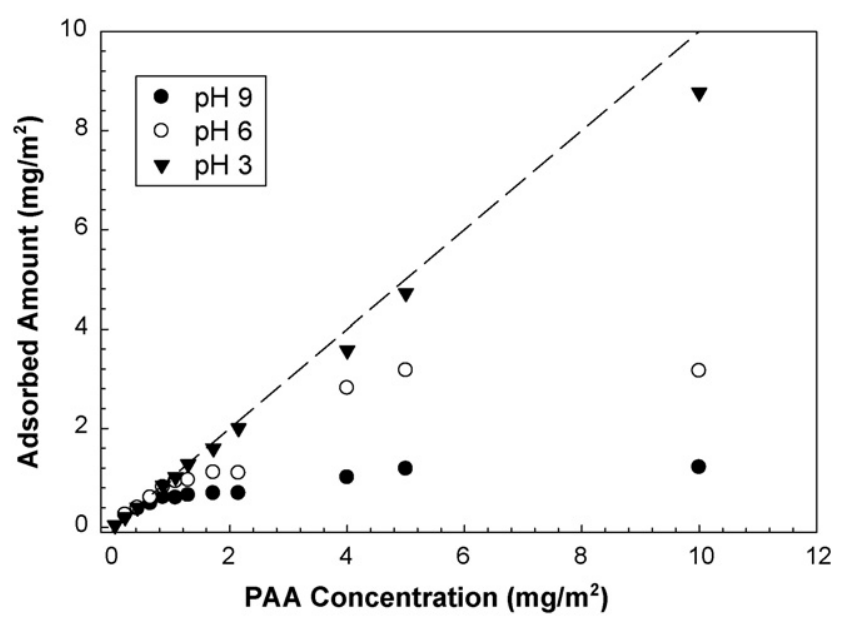

Fig. 4. Adsorption curves of PAA onto PMN surface at $\mathrm{pH} 3,6$ and 9.

Results showed that adsorption of both polyelectrolytes increased with decreasing $\mathrm{pH}$. The adsorption isotherms for PAA at $\mathrm{pH} 3$ can be described as high-affinity type and characterized by a strong adsorption. In contrast, isotherms corresponds to $\mathrm{pH}$ 9 for PAA are low-affinity type with most of the polymer remaining free in solution. Similarly, PAA/PEO comb polymer showed a low-affinity adsorption onto PMN surface at all $\mathrm{pH}$ values. The acidic carboxyl groups in both polyelectrolytes is characterized by a pH-dependent ionization behavior and the ability to exchange with surface hydroxyls bonding with the underlying metal ion in PMN. With the binding of multiple carboxyl groups on the surface this may lead to irreversible high-affinity adsorption.

As it is known, the free energy of adsorption of polyelectrolyte is a balance between the attractive and repulsive interactions. The first term results from the electrostatic attraction between the surface charge group and the opposite charges on the polyelectrolyte, and the contribution of van der Waals interaction between the polyelectrolyte segments and the surface groups. The second originates from entropy loss or osmotic effect [19]. Adsorption of PAA may be dominated by electrostatic interactions between the ionized sites on the polymer and

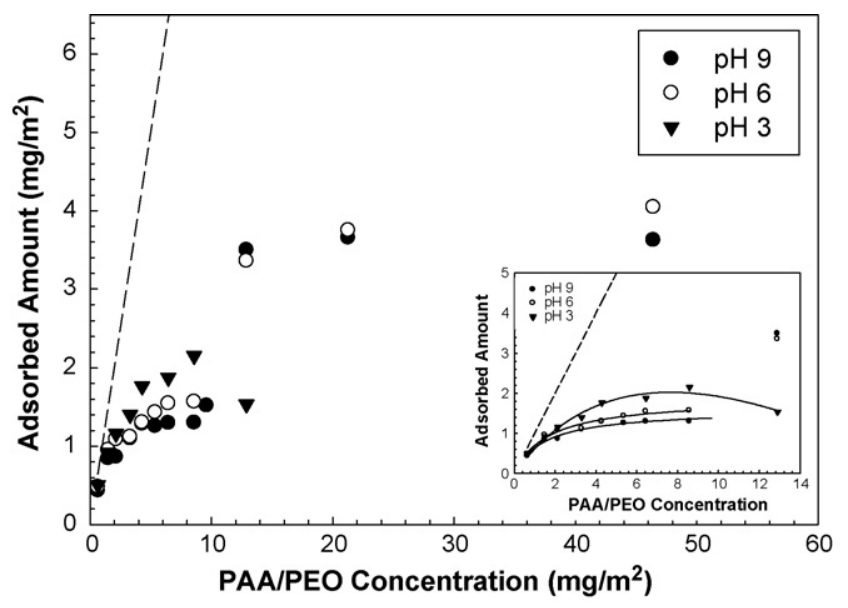

Fig. 5. Adsorption curves of PAA/PEO(2000) onto PMN surface at $\mathrm{pH} \mathrm{3,6}$ and 9. surface charged sites on the PMN. Similarly, interaction between polycarboxylates and metal oxide surfaces have been examined by many researchers, previously.

Cesarano and Aksay [6] examined the adsorption of poly(methacrylic) acid onto alpha alumina surface. According to their results under fully ionized conditions $(\mathrm{pH} 9)$ polyelectrolyte chains adsorb in a relatively flat conformation with each chain covering a large amount of surface area. In this condition polyelectrolyte tails can still extend into the solution even though formation of the loops is inhibited. As the $\mathrm{pH}$ decreased and the ionization approaches to zero the polyelectrolyte chains become uncharged and the formation of the loops in the adsorbed configuration is enhanced [6]. According to the Hackley [7], in the un-dissociated form, PAA adsorption may be controlled by forces dependent on the polymer chain length such as hydrophobic interactions or hydrogen bonding.

Similarly the strong adsorption of PAA onto metal oxides in acidic region have been attributed to the following effects by Paik et al. [20]: (i) ionization is near zero at $\mathrm{pH} 3$ therefore the repulsive interactions between the neighboring carboxyl sites are eliminated. Furthermore the coiled structure permits a denser packing; (ii) solvency will be reduced due to lowionization value and this will force the polymer onto the surface.

Based on the literature [21,22], and the results of this study a possible explanation for the adsorption mechanism of polycarboxylates onto PMN surface is given in the following paragraph:

Below $\mathrm{pH}_{\mathrm{IEP}}$, for adsorption process of the polyelectrolyte with carboxyl groups on positively charged PMN surface the hydrogen bonds and electrostatic interactions are responsible. In $\mathrm{pH} 4.5-9$ range, beside hydrogen bond a chemical interactions between ionized carboxyl groups and $\mathrm{Pb}^{2+}$ and $\mathrm{Mg}^{2+}$ ions present on surface of the oxide. Electrostatic interactions also take place in this $\mathrm{pH}$ range. Above $\mathrm{pH}_{\mathrm{IEP}}$, for adsorption process of polyelectrolytes onto PMN surface only specific interactions are responsible (hydrogen bridge or chemical reactions between $\mathrm{M}^{2+} \mathrm{OH}$ and $-\mathrm{COO}$ groups). In this $\mathrm{pH}$ range there is no electrostatic interactions and therefore, the adsorption of PAA is low.

Fig. 6 shows the adsorption isotherms of PAA/PEO comb polymers having different molecular architecture onto PMN surface at $\mathrm{pH}$ 9. Accordingly, all comb polymers tested in the study showed low-affinity type adsorption onto PMN surface. Adsorption behavior was almost the same for all the comb polymers although their molecular weight and the acid:ester ratios were not identical. Therefore, it is concluded that adsorption of PAA/PEO comb polymers was not very much dependent on the length of the PEO group.

On the other hand, a multilayer adsorption was observed in the presence of comb polymers. The reason of the possible multilayer adsorption can be explained by the previous theory of Dobbie et al [23]. Accordingly, they drawn attention to the interaction between the carboxyl groups and the PEO and claimed that there is hydrogen bonding between the $-\mathrm{OH}$ groups in the surface carboxyl groups of the polystyrene lattices and the ether oxygen of the PEO group. Similarly, in our case it may be possible to consider an interaction between the PEO groups of the 


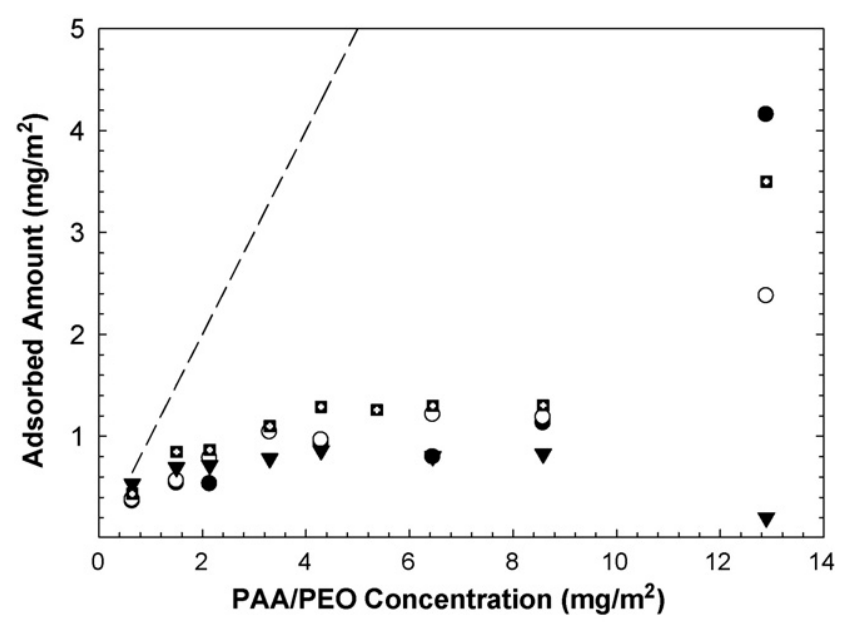

- PAA/PEO(1000), acid:ester (7:1)

O PAA/PEO(1000), acid:ester (3:1)

$\nabla$ PAA/PEO(400), acid:ester (3:1)

- PAA/PEO(2000), acid:ester (7:1)

Fig. 6. Adsorption curves of PAA/PEO comb polymers having different molecular architecture onto PMN surface, $\mathrm{pH} 9$.

adsorbed comb polymer and the PAA group of the excess free comb polymer in suspension [23].

In the current study, it is also important to know that there is no adsorption between the PEO groups of the comb polymer and the PMN surface because PEO adsorbs only on surfaces with strong Bronsted acid sites, such as $\mathrm{MoO}_{3}, \mathrm{~V}_{2} \mathrm{O}_{5}$ and $\mathrm{SiO}_{2}$. For materials with IEP greater than $\mathrm{SiO}_{2}$ adsorption of PEO does not occur [24]. Therefore there is a low possibility for an interaction between the PMN and PEO groups of the comb polymer.

Other possible explanation for the multilayer adsorption observed at high polymer concentrations can be attributed to the low-surface area of PMN in highly concentrated polymer solutions. Zhonghua and co-workers [22] investigated the effect of solids loading on the adsorption density of PAA on $\mathrm{Al}_{2} \mathrm{O}_{3}$. Results showed that addition of a few alumina particles to a solution of high polymer concentration may in fact "seed" the precipitation or multilayer adsorption of PAA onto alumina. Therefore, apparent adsorption density greatly increased at low solids concentrations. As more solids are added however, the surface area for adsorption was no longer limited and the adsorption density reached [22].

In this study the effect of monovalent and divalent salt additions on the adsorption behavior of PAA and PAA/PEO comb polymers was also studied as a function of polymer concentration. Figs. 7 and 8 depict the effect of background electrolytes on the adsorption of PAA/PEO and PAA, respectively. According to Fig. 7 in the presence comb polymer both monovalent and divalent salts caused a slight increase in the adsorbed amount. However, the effect was more significant in the case of PAA. Because the background salt has an electrostatic shielding effect between the negatively charged sites on the PAA thereby causing the chains behave more like uncharged polymers and enhancing the development of the loops [6].

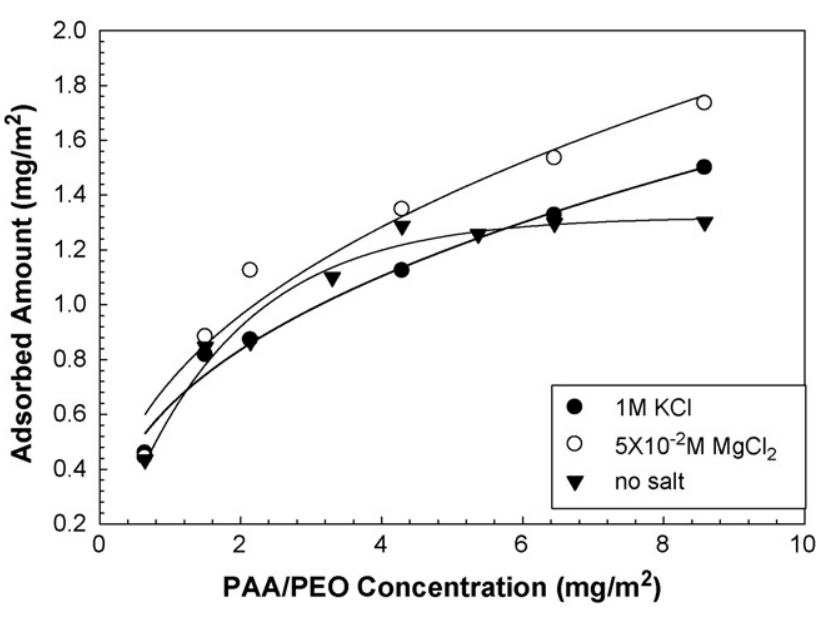

Fig. 7. Effect of monovalent and divalent salt additions on the adsorption behavior of PAA/PEO(2000) onto PMN surface at $\mathrm{pH} 9$.

Results revealed that for both polyelectrolytes under investigation the adsorption amount was slightly higher in the presence of $\mathrm{MgCl}_{2}$ compared to the $\mathrm{KCl}$. Because adsorption of PAA can be strongly affected by the presence of multivalent ions. Vermohlen et al. [25] investigated the effect of $\mathrm{Ca}^{2+}$ on the adsorption of acrylic based polyelectrolytes onto oxide surfaces. It was found that the plateau value of the adsorption isotherms is greatly increased by $\mathrm{Ca}^{2+}$ ions. This is because multivalent cations can bind to the carboxyl functional groups of the polyelectrolyte to form PAA-cation complexes $\left(\mathrm{R}-\mathrm{COO}^{-}-\right.$ $\left.\mathrm{M}^{2+}{ }_{-}{ }^{-} \mathrm{OOC}-\mathrm{R}\right)$ in solution. This complexation can promote the adsorption by lowering the net charge of the polyelectrolyte or by decreasing the solubility of the complex in solution [26].

Similarly, effect of $\mathrm{Ca}^{2+}$ ions on the adsorption behavior of PAA onto alumina surface was examined by Dupont et al. [27]. They found that in alkaline media, PAA adsorption strongly depends on the calcium ion concentration, however, in acidic media only a slight influence of the calcium ions was found. Due to the electrostatic effects, the complexation ratio was slightly higher in the adsorption layer than in the bulk of the solution [27].

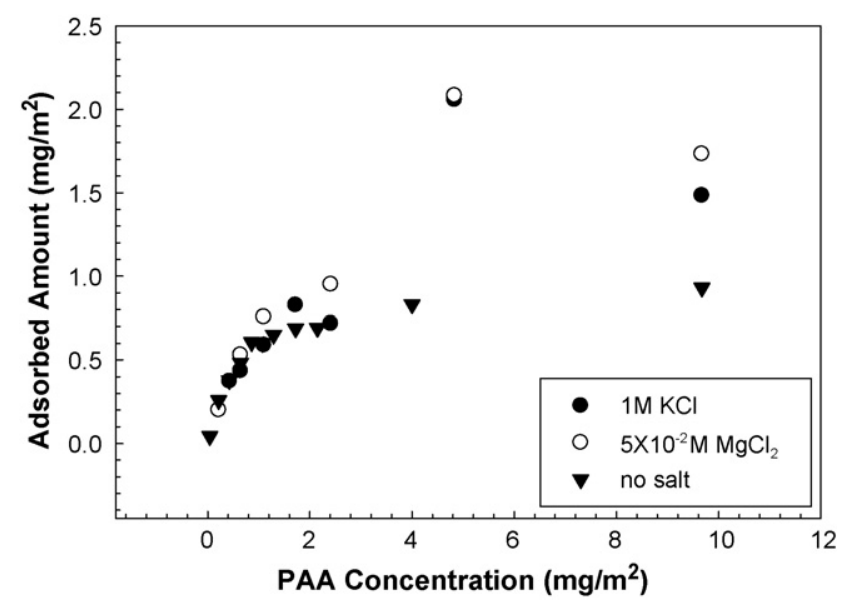

Fig. 8. Effect of monovalent and divalent salt additions on the adsorption behavior of PAA onto PMN surface at $\mathrm{pH} 9$. 


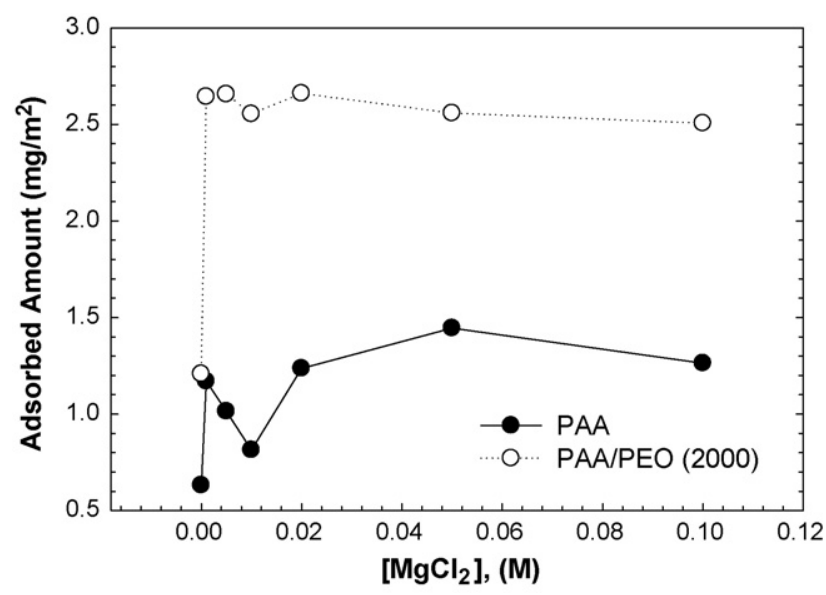

Fig. 9. Effect of $\mathrm{MgCl}_{2}$ concentration on the adsorption behavior of PAA $\left(1.1 \mathrm{mg} / \mathrm{m}^{2}\right)$ and PAA/PEO(2000) $\left(4.2 \mathrm{mg} / \mathrm{m}^{2}\right)$ at $\mathrm{pH} 9$.

Sun and co-workers investigated the effects of magnesium ions on the adsorption of PAA onto alumina. They found that the addition of $\mathrm{Mg}^{2+}$ ions has a strong effect on the adsorption of PAA and maximum adsorbed amount increases five times with increasing $\mathrm{MgCl}_{2}$ concentration [26].

In the current study we observed that in the presence of PAA, addition of $\mathrm{MgCl}_{2}$ increased the adsorption nearly 2.6 times. It is possible to attribute this increase to the complex formation between PAA and $\mathrm{Mg}^{2+}$ ions. On the other hand, the increase in the adsorption of PAA/PEO onto PMN in the presence of monovalent or divalent salt may be attributed to the decrease in the electrostatic forces rather than complex formation with the divalent metal ions in solution.

It is also important to know that complexation promotes adsorption but the effect is very sensitive to the ratio of cation to dissociated carboxylic acid groups in the polyelectrolyte. Therefore, increasing the PAA concentration beyond the optimal ratio decreases the adsorbed amount. This is because less complexed PAA has a lower driving force for adsorption [26]. Dupont et al. [27] found a decrease in the adsorption isotherms as PAA concentration increases at constant calcium concentration, and it was due to decrease in the complexation ratio. Fig. 9 shows the effect of $\mathrm{MgCl}_{2}$ concentration on the adsorption amount of polyelectrolytes onto PMN surface. According to the graph highest adsorption amount were obtained in the presence of $2 \times 10^{-2}$ and $5 \times 10^{-2} \mathrm{M} \mathrm{MgCl}_{2}$ for PAA/PEO and PAA, respectively.

\subsection{Turbidity measurements}

In the study, turbidity measurements were carried out on dilute polymer solutions of varying $\mathrm{pH}$ and salt to investigate the complexation between the metal ions and the PAA. Figs. 10 and 11 show the turbidity values of polymer solutions as a function of ionic strength and $\mathrm{pH}$. The stable polymer solutions characterized in which the polymeric species remain well solvated and are transparent. Therefore, high turbidity values correspond to the solutions having low stability. In

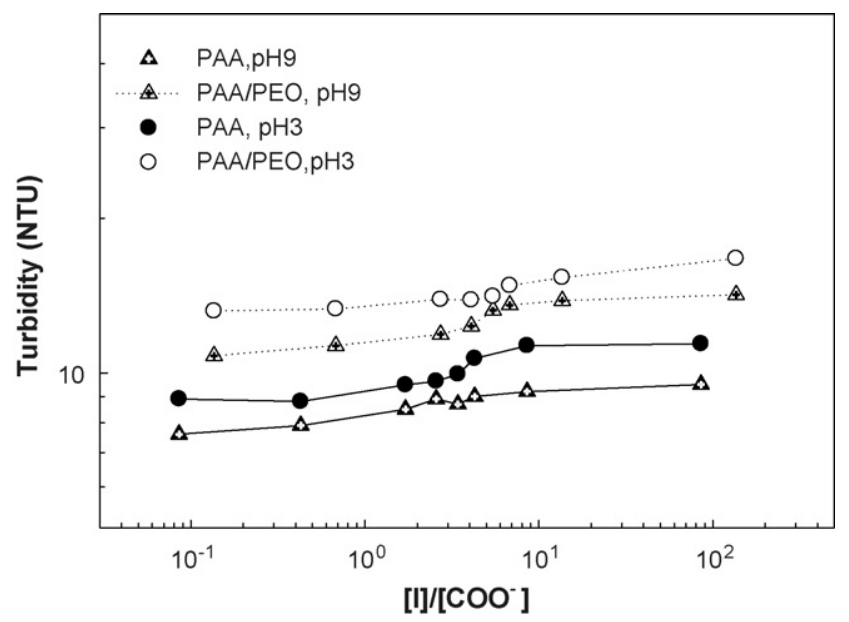

Fig. 10. Effect of $\mathrm{KCl}$ on the turbidity of PAA and PAA/PEO(2000) solutions as a function of ionic strength at $\mathrm{pH} 3$ and 9 . Note, $[\mathrm{I}]=1 / 2 \sum n_{i} z_{i}^{2}$ due to added salt only, $\left[\mathrm{COO}^{-}\right]=0.07 \mathrm{M}$, and $0.011 \mathrm{M}$ for PAA and PAA/PEO(2000), respectively.

other words, as the turbidity increases the solution stability decreases.

According to Fig. 10 in the presence of monovalent salt $(\mathrm{KCl})$ both PAA and PAA/PEO solutions remained stable over a broad range of $[\mathrm{I}] /\left[\mathrm{COO}^{-}\right]$and in acidic and basic $\mathrm{pH}$ regions. However, in the presence of divalent salt $\left(\mathrm{MgCl}_{2}\right)$ the stability of PAA solutions depended on $\mathrm{pH}$ and salt concentration. At low $\mathrm{pH}$ values where PAA is slightly ionized PAA solutions remained stable over a broad range of $[\mathrm{I}] /\left[\mathrm{COO}^{-}\right]$values. On the other hand in fully ionized conditions ( $\mathrm{pH}$ 9) PAA solutions were only stable in the range $0-2 \times 10^{-2} \mathrm{M} \mathrm{MgCl}_{2}$. At higher salt concentrations PAA start to make complexes with $\mathrm{Mg}^{2+}$ ions in solution.

To explain the findings of the current study the dominant role of PEO teeth is illustrated schematically based on the previous study of Kirby and Lewis [11] in Fig. 12. PEO teeth provide two important functions to prevent deleterious effects of the dissolved ions on PMN suspension stability. First, charge neutral

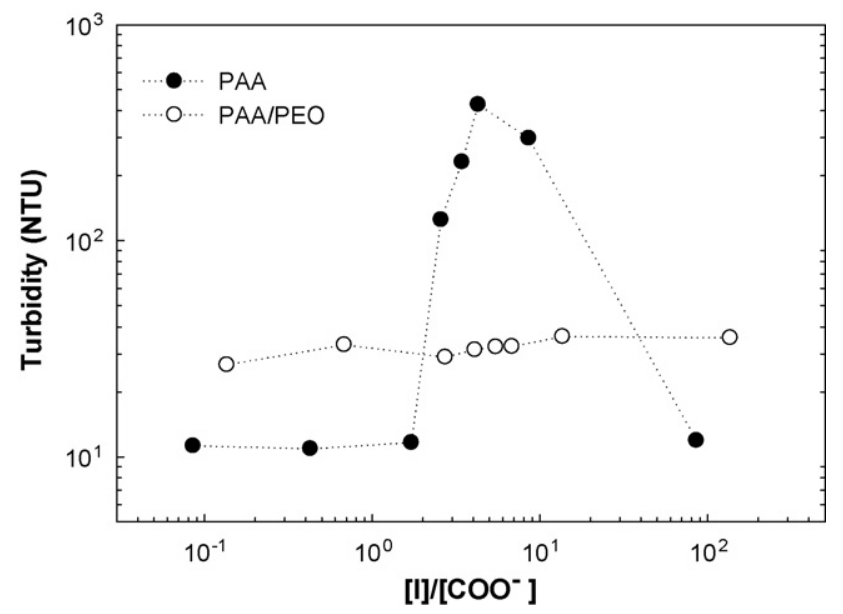

Fig. 11. Effect of $\mathrm{MgCl}_{2}$ on the turbidity of PAA and PAA/PEO (2000) solutions as a function of ionic strength at $\mathrm{pH} 9$. Note, $[\mathrm{I}]=1 / 2 \sum n_{i} z_{i}^{2}$ due to added salt only, $\left[\mathrm{COO}^{-}\right]=0.07 \mathrm{M}$, and $0.011 \mathrm{M}$ for PAA and PAA/PEO(2000), respectively. 


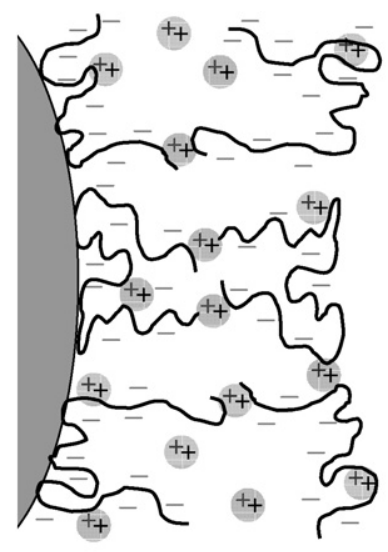

(a)
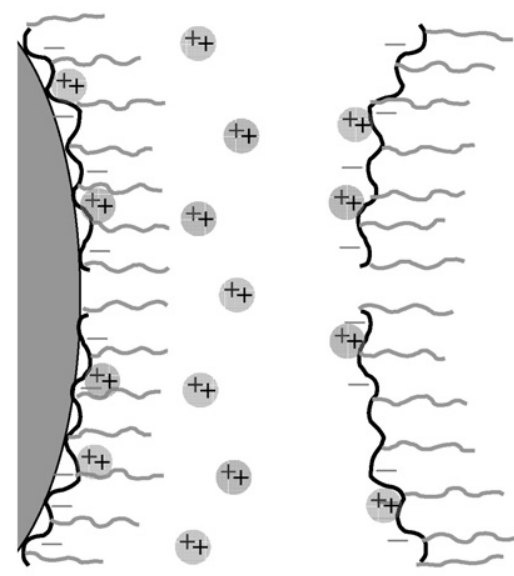

(b)

Fig. 12. Schematic illustrations of (a) interaction between PAA and the multivalent ions in solution and (b) shielding from ion bridging interactions provided by the PEO teeth. Adapted from Kirby and Lewis [11].

PEO teeth shield the PAA backbone from ion bridging interactions with the multivalent counter ions in solution. Second, the charge neutral PEO teeth do not experience large conformational changes with changes in $\mathrm{pH}$ or ionic strength [11]. As a result, the conformation of the PAA/PEO layers adsorbed on the PMN surfaces remains nearly constant as the ionic strength increases.

\section{Conclusions}

According to the results of the current work following conclusions can be drawn: polycarboxylate adsorption onto PMN increases with decreasing $\mathrm{pH}$ and increasing salt concentration. Furthermore, saturation adsorption results in partial charge reversal and a negative zeta potential. Adsorption of high molecular weight comb polymers onto PMN powder can be described by the low surface coverage. $\mathrm{MgCl}_{2}$ increased the adsorption nearly two times for both PAA and PAA/PEO. The increase in the adsorption of PAA/PEO onto PMN in the presence of monovalent or divalent salt was attributed to the decrease in the electrostatic forces rather than complex formation with the divalent metal ions in solution. In contrast, the resultant effect was directly related with the complex formation in the case of PAA.

\section{Acknowledgements}

Zeta potential measurements were performed at University of Illinois at Urbana, Champaign, USA. Invaluable advice and support of Prof. Dr. Jennifer A. Lewis is gratefully acknowledged. The financial support and bursary by The Scientific and Technological Research Council of Turkey (Tubitak NATO-A2) and State Planning Organization of Turkey (DPT) is acknowledged.

\section{References}

[1] S. Fengbing, L. Qiang, Z. Haisheng, L. Chunhong, Z. Shix, S. Dezhong, Phase formation and transitions in the lead magnesium niobate-lead titanate system, Mater. Chem. Phys. 83 (2004) 135-139.

[2] A. Brailenau, A. Ianculescu, M. Zaharescu, I. Pasuk, S. Preda, J. Madarasz, G. Pokol, Phase formation study of La-modified lead magnesium niobate, Key Eng. Mater. 264-268 (2004) 1309-1312.

[3] H.S. Tzou, H.J. Lee, S.M. Arnold, Smart materials precision sensors/actuators, smart structures, and structronic systems, Mech. Adv. Mater. Struct. 11 (2004) 367-393.

[4] J.A. Lewis, Colloidal processing of ceramics, J. Am. Ceram. Soc. 83 (2000) 2341-2359.

[5] H. Guldberg-Pedersen, L. Bergstrom, Stabilizing ceramic suspensions using anionic polyelectrolytes: adsorption kinetics and interparticle forces, Acta Mater. 48 (2000) 4563-4570.

[6] J. Cesarano III, I.A. Aksay, Stability of aqueous $\alpha$-alumina suspensions with poly(methacrylic acid) polyelectrolyte, J. Am. Ceram. Soc. 71 (1988) 250-255.

[7] V.A. Hackley, Colloidal processing of silicon nitride with poly(acrylic acid): adsorption and electrostatic interactions, J. Am. Ceram. Soc. 80 (1997) 2315-2325.

[8] C.-C. Li, J.-H. Jean, Interaction between dissolved $\mathrm{Ba}^{2+}$ and PAA-NH dispersant in aqueous barium titanate suspensions, J. Am. Ceram. Soc. 85 (2002) 1449-1455.

[9] H.G. Kirby, D. Harris, Q. Li, J.A. Lewis, Polyacrylic acid-polyethylene oxide comb polymer effects on $\mathrm{BaTiO}_{3}$ nanoparticle suspension stability, J. Am. Ceram. Soc. 87 (2004) 181-186.

[10] J.H. Adair, J. Crampo, M.M. Mandanas, E. Suvaci, The role of material chemistry in processing $\mathrm{BaTiO}_{3}$ in aqueous suspensions, J. Am. Ceram. Soc. 89 (2006) 1853-1860.

[11] G.H. Kirby, J.A. Lewis, Comb polymer architecture effects on rheological property evolution of concentrated cement suspensions, J. Am. Ceram. Soc. 87 (2004) 1643-1652.

[12] C.P. Whitby, P.J. Scales, F. Grieser, T.W. Healy, G. Kirby, J.A. Lewis, C.F. Zukoski, PAA/PEO comb polymer effects on rheological properties and interparticle forces in aqueous silica suspensions, J. Colloid Interf. Sci. 81 (2003) 262-274.

[13] A.M. Deliormanlı, J.A. Lewis, E. Çelik, M. Polat, Colloidal behavior of aqueous lead magnesium niobate suspensions in the presence of PAA/PEO comb polymers, presented at 10th International Conference of the European Ceramic Society, Berlin, June 1721, 2007.

[14] A.M. Deliormanlı, E. Çelik, M. Polat, The isoelectric point of lead magnesium niobate, J. Am. Ceram. Soc. 90 (2007) 3314-3317.

[15] A. Neubrand, R. Lindner, P. Hoffmann, Room temperature solubility behaviour of barium titanate in aqueous media, J. Am. Ceram. Soc. 83 (2000) 860-864.

[16] U. Paik, A.V. Hackley, Influence of solids concentration on the isoelectric point of aqueous barium titanate, J. Am. Ceram. Soc. 83 (2000) 2381-2384.

[17] A. Shair, D. Andelman, Polyelectrolyte adsorption: chemical and electrostatic interactions, Phys. Rev. E 70 (2004) 061804.

[18] J.F. Joanny, Polyelectrolyte adsorption and charge inversion, Eur. Phys. J. B 9 (1999) 117-122.

[19] H. Bouhamed, S. Boufi, A. Magnin, Alumina interaction with AMPS-PEG random copolymer II. Stability and rheological behvaiour, Colloids Surf. A: Physicochem. Eng. Aspect. 253 (2005) 145-153. 
[20] U. Paik, A.V. Hackley, J. Lee, S. Lee, Effect of poly(acrylic acid) and poly(vinyl alcohol) on the solubility of colloidal $\mathrm{BaTiO}_{3}$ in an aqueous medium, J. Mater. Res. 8 (2003) 5.

[21] S. Chibowski, M. Paszkiewicz, Polyacrylic acid (PAA) adsorption on alumina surface: influence of sodium dodecyl sulfide (SDS) on adsorption in PAA-SDS- $\mathrm{Al}_{2} \mathrm{O}_{3}$ system, Physicochem. Problems Miner. Process. 40 (2006) 175-184.

[22] P. Zhonghua, A. Campbell, P. Somasundaran, Polyacrylic acid adsorption and conformation in concentrated alumina suspensions, Colloids Surf. A. Physicochem. Eng. Asp. 191 (2001) 71-78.

[23] J.W. Dobbie, R. Evans, D.V. Gibson, J.B. Smitham, D.H. Napper, Enhanced steric stabilization, J. Colloid Interf. Sci. 45 (1973).
[24] J. Sindel, N.S. Bell, W.M. Sigmund, Electrolyte effects on nonionic steric layers: bis-hydrophilic PMAA-PEO diblock copolymers, adsorbed on barium titanate, J. Am. Ceram. Soc. 82 (1999) 2953-2957.

[25] K. Vermohlen, H. Lewandowski, H.D. Narres, M.J. Schwuger, Adsorption of polyelectrolytes onto oxides-the influence of ionic strength, molar mass and $\mathrm{Ca}^{2+}$ ions, Colloids Surf. A.: Physicochem. Eng. Aspect. 163 (2000) $45-53$.

[26] J. Sun, L. Bergstrom, L. Gao, Effect of magnesium ions on the adsorption of polyacrylic acid onto alumina, J. Am. Ceram. Soc. 84 (2001) 2710-2712.

[27] L. Dupont, A. Foissy, R. Mercier, B. Mottet, Effect of calcium ions on the adsorption of polyacrylic acid onto alumina, J. Colloid Interf. Sci. 161 (1993)455-464. 